\title{
Synthesis of Various Esters of Diclofenac (NSAIDs) as Pro-Drugs and their Biological Evaluation
}

\author{
S. B. SURYAWANSHI, HASSAN.A.OSMAN, Y.I.SHAIKH and G. M. NAZERUDDIN* \\ Department of Chemistry (P.G. \& Research Centre), \\ Poona College of Arts, Science \& Commerce, Pune, India \\ gmnazeruddin@yahoo.co.in
}

Received 10 October 2013 / Accepted 13 November 2013

\begin{abstract}
Non-steroidal anti-inflammatory drugs (NSAIDs) commonly used for the treatment of chronic inflammatory diseases suffering from several undesired side effects, the most important being gastrointestinal (GI) irritation and ulceration. The pro-drug designing is one of the several strategies used to overcome this drawback. The rationale behind the prodrug concept is to achieve temporary blockade of the free carboxylic group present in the NSAIDs till their systemic absorption. Five esters of diclofenac were synthesized with different alcohols and all the compounds were evaluated for anti-inflammatory activity by Carrageenan Induced Rat hand Paw method and surprisingly all of them showed quite appreciable anti-inflammatory activity.
\end{abstract}

Keywords: Anti-inflammatory activity, Various esters of Diclofenac, Gastrointestinal irritation

\section{Introduction}

Non-steroidal anti-inflammatory drugs (NSAIDs) are commonly used for the treatment of chronic inflammatory diseases such as arthritis. Prolonged administration of these drugs exhibits several undesired side effects, most important are gastrointestinal irritation and ulceration $^{1,2}$. There is enough evidence that inhibition of Cox-I rather than that of Cox-II underlies gastric ulcer formation ${ }^{3}$. But initial enthusiasm for selective Cox-II inhibitor as safer NSAIDs has failed due to emergence of serious cardiovascular side effect on long term use $^{4}$. The Pro-drug designing ${ }^{5}$ is one of the several strategies used to overcome this drawback. Considerable attention has been focused on the development of bioreversible derivatives, such as pro-drugs, to temporarily mask the acidic group of NSAIDs as a promising means of reducing or abolishing the GI toxicity due to the local action mechanism. Pro-drugs are pharmacologically inactive derivatives of active agents, which undergo chemical and/or enzymatic biotransformation resulting in the release of active drug after administration. The metabolic product (i.e. parent drug) subsequently elicits the desired pharmacological response ${ }^{4,5}$. Most pro-drugs of NSAIDs have been prepared by derivatization of the carboxyl group. The esters have dominated pro drug research because they have the ideal characteristic of exhibiting reasonable in vitro chemical stability which 
allows them to be formulated with adequate shelf lives. In addition, by virtue of their ability to function as esterase substrates, esters are suitably labile, in $v i v o^{6,7}$. With this aim different esters of diclofenac were synthesized and evaluated. The reaction is depicted in Scheme 1.

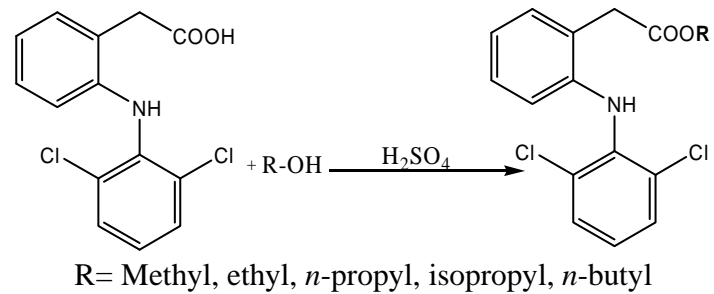

\section{Experimental}

Scheme 1. Synthesis of ester compounds (a-e)

All reagents were purchased from Merck and Loba and used without further purification. Melting points were measured in open capillary and are uncorrected. The products were characterized by IR spectra, ${ }^{1} \mathrm{H}$ NMR. IR spectra were recorded on Perkin-Elmer FT-IR-1710 instrument. ${ }^{1} \mathrm{H}$ NMR was recorded on BrukerMSL-300 MHz instrument using TMS as an internal standard.

\section{Methods}

\section{General procedure for the preparation of various esters of diclofenac}

Methyl, ethyl, $n$-propyl, isopropyl and $n$-butyl esters of diclofenac were synthesized using direct esterification method. Diclofenac $(0.004 \mathrm{~mol})$ and respective alcohol $(0.25 \mathrm{~mol})$ were taken in round bottom flask fitted with reflex condenser. $1 \mathrm{~mL}$ of conc. Sulphuric acid was added gradually to this reaction mixture. The reaction mixture was refluxed with continuous stirring at $80 \mathrm{C}$. until the esterification was completed. The progress of the reaction was monitored by TLC. After completion of the reaction excess alcohol was removed by distillation under reduced pressure. Crude product was dissolved in $30, \mathrm{~mL}$ dichloromethane and was washed by $30 \mathrm{~mL}$ water and dried over anhydrous sodium sulphate followed by filtration and solvent was removed under vacuum to furnish the crude product which was purified by column chromatography 5\% ethyl acetate in pet ether as an eluent.

\section{Carrageenan-induced paw edema}

\section{Purpose and rationale}

Among the many methods used for screening of anti-inflammatory drugs, one of the most commonly employed techniques is based upon the ability of such agents to inhibit the edema produced in the hind paw of the rat after injection of a phlogistic agent. Many phlogistic agents (irritants) have been used such as brewer's yeast, formaldehyde etc. the effect can be measured in several ways. Many methods have been described to measure the paw volume by simple and less accurate and by more sophisticated electronically devised methods.

\section{Procedure}

Male or female Wistar Albino rats with body weight 100-200 g were taken. The animals were starved overnight before start of the study. The animals dosed with $10 \mathrm{mg} / \mathrm{kg} \mathrm{b.w.} \mathrm{of}$ the test drugs and control received the same volume of water.

After $30 \mathrm{~min} .0 .05 \mathrm{~mL}$ of $1 \%$ solution of carrageenan was injected into the plantar side of the left hind paw. The paw was marked with ink at the level of lateral malleolus and immersed in mercury up to the mark to determine the paw volume. Similarly after $30 \mathrm{~min}, 1 \mathrm{~h}$ and $2 \mathrm{~h}$, the paw volume was measured. 


\section{Evaluation}

The volumes were obtained in $\mathrm{mL}$ and the average (mean) values were obtained by the statistical analysis. The statistical analysis was performed by using One way ANOVA followed by Dunnet's test.

\section{Characterization}

Methyl2-(2-(2,6-dichlorophenylamino)phenyl)acetate: Yield=80\%, M.P=97-99 ${ }^{0} \mathrm{C}$, IR(KBr) 3364,3019,1726,1578,1507,1454,1216,1151,751 cm $\mathrm{cm}^{-1}{ }^{1} \mathrm{H}$ NMR $\left(\mathrm{CDCl}_{3}\right) \mathrm{d} 1.54$ (s,NH), 3.69 $\left(\mathrm{S}, \mathrm{OCH}_{3}\right), 3.95\left(\mathrm{~s}, \mathrm{CH}_{2}\right), 6.66-7.50(\mathrm{~m}, \mathrm{Ar}-7 \mathrm{H})$.

Ethyl2-(2-(2,6-dichlorophenylamino)phenyl) acetate: Yield $=74 \%$, M.P $=68-70{ }^{0} \mathrm{C}$, IR(KBr) 3318,2981,1721,1578,1507,1454,1370,1296, 1149,1028,771,746 $\mathrm{cm}^{-1},{ }^{1} \mathrm{H}$ NMR, $\left(\mathrm{CDCl}_{3}\right)$ 1.31(t, $\left.\mathrm{CH}_{3}\right), 1.52(\mathrm{~s}, \mathrm{NH}), 3.83\left(\mathrm{~s}, \mathrm{OCH}_{2}\right), 4.23\left(\mathrm{q}, \mathrm{CH}_{2}\right), 6.55-7.38(\mathrm{~m}, \mathrm{Ar}-7 \mathrm{H})$.

n-Propyl 2-(2-(2,6-dichlorophenylamino)phenyl)acetate: Yield=78\%,M.P=132-134 ${ }^{0} \mathrm{C}$, IR(KBr)3448, 2916,1730,1612,1469,1359, 1240,1195,786 cm ${ }^{-1},{ }^{1} \mathrm{H}$ NMR $\left(\mathrm{CDCl}_{3}\right) 1.25$ $\left(\mathrm{t}, \mathrm{CH}_{3}\right), 1.60(\mathrm{~s}, \mathrm{NH}), 2.1\left(\mathrm{~m}, \mathrm{CH}_{2}\right), 3.81\left(\mathrm{~s}, \mathrm{OCH}_{2}\right), 4.1\left(\mathrm{~m}, \mathrm{CH}_{2}\right), 6.4-7.6(\mathrm{~m}, \mathrm{Ar}-7 \mathrm{H})$.

Isopropyl2-(2-(2,6-dichlorophenylamino)phenyl) acetate: Yield=82\%, M.P =88-90 ${ }^{0} \mathrm{C}$, IR(KBr) 3319,2983,1716,1578,1506,1454,1255,1270,1105,758 $\mathrm{cm}^{-1},{ }_{1}^{1} \mathrm{H}$ NMR $\left(\mathrm{CDCl}_{3}\right) 1.21$ $\left(\mathrm{d}, 2 \mathrm{CH}_{3}\right), 1.52(\mathrm{~s}, \mathrm{NH}), 3.73\left(\mathrm{~s}, \mathrm{CH}_{2}\right), 5.02(\mathrm{~m}, \mathrm{CH}), 6.49-7.31(\mathrm{~m}, \mathrm{Ar}-7 \mathrm{H})$.

n-Butyl 2-(2-(2,6-dichlorophenylamino)phenyl)acetate: Yield=68\%,M.P =138-140 ${ }^{\circ} \mathrm{C}$ IR (KBr)3429,2926,1720,1563,1506,1454,1149,756 $\mathrm{cm}^{-1},{ }^{1} \mathrm{H}$ NMR $\left(\mathrm{CDCl}_{3}\right)$ 0.82(t, $\left.\mathrm{CH}_{3}\right), 1.18-$ $1.50\left(\mathrm{~m}, 2 \mathrm{CH}_{2}\right), 2.10(\mathrm{~s}, \mathrm{NH}), 3.73\left(\mathrm{~s}, \mathrm{CH}_{2}\right), 4.07\left(\mathrm{t}, \mathrm{OCH}_{2}\right), 6.5-7.4(\mathrm{~m}, \mathrm{Ar}-7 \mathrm{H})$.

\section{Results and Discussion}

In the present research work five esters of diclofenac were synthesized as mentioned in the Scheme 1 and experimental work. All these compounds were tested for their purity by TLC and melting point. The structures of these esters were confirmed by IR NMR. All these were found to be satisfactory. The synthesized compounds were evaluated for anti-inflammatory activity by Paw edema method using carragenan. The inflammation was measured by PM these compounds were given by IM. There was significant reduction in the inflammation. All the esters showed promising anti-inflammatory activity. The readings were calculated by One way ANOVA followed by Dunnet's t test. Diclofenac is used as standard drug, with suitable molecular modification and manipulations, these compounds may be excellent antiinflammatory agents in future.

Table 1. Effect of various synthesized compounds on carrageenan -induced paw oedema in Rats in different time

\begin{tabular}{ccccc}
\hline Treatment and dose & \multicolumn{4}{c}{ Oedema volume } \\
\cline { 2 - 5 } $100 \mathrm{mg} / \mathrm{mL}$ & $0 \mathrm{~h}$ & $0.5 \mathrm{~h}$ & $1 \mathrm{~h}$ & $2 \mathrm{~h}$ \\
\hline $\mathbf{a}$ & $1.35 \pm 0.08^{*}$ & $1.65 \pm 0.06^{*}$ & $1.45 \pm 0.02^{*}$ & $1.32 \pm 0.11^{*}$ \\
$\mathbf{b}$ & $1.27 \pm 0.08^{*}$ & $1.65 \pm 0.10^{*}$ & $1.20 \pm 0.04^{*}$ & $1.10 \pm 0.04^{*}$ \\
$\mathbf{c}$ & $1.37 \pm 0.08^{*}$ & $1.57 \pm 0.04^{*}$ & $1.20 \pm 0.04^{*}$ & $1.10 \pm 0.04^{*}$ \\
$\mathbf{d}$ & $1.45 \pm 0.06^{*}$ & $1.57 \pm 0.07^{*}$ & $1.32 \pm 0.04^{*}$ & $1.15 \pm 0.06^{*}$ \\
$\mathbf{e}$ & $1.25 \pm 0.06^{*}$ & $1.72 \pm 0.07^{*}$ & $1.50 \pm 0.05^{*}$ & $1.20 \pm 0.04^{*}$ \\
Diclofenac & $1.27 \pm 0.09$ & $1.90 \pm 0.04^{*}$ & $1.60 \pm 0.04^{*}$ & $1.27 \pm 0.04^{*}$ \\
\hline \multicolumn{5}{c}{$p<0.05$ SEM $=$ Standard Error to Mean }
\end{tabular}




\section{Conclusion}

Non-steroidal anti-inflammatory drugs commonly used for the chronic inflammatory diseases suffer from several side effects, out of which GI irritation and ulceration are most common. Prodrug designing is one of the strategies used to overcome this limitation. However, the prodrugs developed essentially need to be characterized as evident by their altered profile, one of the examples being frequent poor solubility. This area of research has rendered the clinically useful drugs as safer choice by limiting their undesirable characteristics and improving their therapeutic proficiency. The scope is unlimited with vast potential, and it is expected to be safer.

\section{Acknowledgement}

Authors are thankful to Anjuman Khairul-Islam Trust Mumbai for their financial assistance.

\section{References}

1. Perez G. S, Garcia R L A, Rainford, Duque O A and Ris R J, Arc Inter Med., 1996, 156, 2433-2439.

2. Polonia J J Cardiol, Cardiology, 1997, 88, 47-51.

3. Meade E A, Smith W L and De Witt D L, J Biol Chem., 1993, 268, 6610-6614

4. Renter B K, Asfaha S, Buret-n Sharkey K A and Wallace J A, J Chin Invest., 1996, 98, 2076-2085.

5. Parmeshwari K Halen, Prashant R Murumkar, Rajani Giridhar and Mange Ram Yadav, Mini-Rev Med Chem., 2009, 9, 124-139

6. Bundgaard H, Adv Drug Deliv Rev., 1989, 3(1), 39-65;

DOI:10.1016/0169-409X(89)90004-5

7. Bundgaard H, In: Design of Prodrugs; Bundgaard, H. Ed., Elsevier, New York, Plenum Press, 1986, 49-68. 